\title{
Muséologies
}

Les cahiers d'études supérieures

muséologies

\section{Carnet des Rencontres indisciplinaires}

\section{Myriam Suchet}

Volume 9, numéro 1, 2018

Monde des Arts / Arts des mondes

URI : https://id.erudit.org/iderudit/1052631ar

DOI : https://doi.org/10.7202/1052631ar

Aller au sommaire du numéro

Éditeur(s)

Association Québécoise de Promotion des Recherches Étudiantes en

Muséologie (AQPREM)

ISSN

1718-5181 (imprimé)

1929-7815 (numérique)

Découvrir la revue

Citer ce compte rendu

Suchet, M. (2018). Compte rendu de [Carnet des Rencontres indisciplinaires].

Muséologies, 9(1), 113-133. https://doi.org/10.7202/1052631ar

Tous droits réservés (C Association Québécoise de Promotion des Recherches Étudiantes en Muséologie (AQPREM), 2018
Ce document est protégé par la loi sur le droit d'auteur. L’utilisation des services d'Érudit (y compris la reproduction) est assujettie à sa politique d'utilisation que vous pouvez consulter en ligne.

https://apropos.erudit.org/fr/usagers/politique-dutilisation/ 
Carnet un

Carnet des Rencontres indisciplinaires

Myriam Suchet 
Myriam Suchet est maître de conférence à l'Université Paris 3 - Sorbonne Nouvelle, où elle dirige le Centre d'études québécoises depuis sa création en 2012 . Elle est titulaire d'un doctorat in Humanities mené entre les universités Lille 3 et Concordia (prix de la meilleure thèse en cotutelle France-Québec et Médaille d'or du Gouverneur général du Canada en 2011). Elle a publié trois ouvrages: Indiscipline! Tentatives d'UniverCité à l'usage des littégraphistes, artistechniciens et autres philopraticiens (Montréal: Nota Bene, 2016), L'imaginaire hétérolingue. Ce que nous apprennent les textes à la croisée des langues (Paris: Classiques Garnier, 2014) et Outils pour une traduction postcoloniale (Paris: Archives contemporaines, 2009). D'autres articles sont disponibles en ligne, notamment dans les revues Cousins de personne, Quaderna et LHT, pour laquelle elle a coordonné avec Samia Kassab le dossier «La langue française n'existe pas». Elle travaille notamment à déployer un imaginaire hétérolingue qui bouscule « la langue " et à penser-pratiquer la recherche comme une forme relationnelle d'action-création. 
DAMASIO, Alain. La Horde du Contrevent. Paris: La Volte, 2004, p. 39 :

Les aerudits affirment que la neuvième forme [du vent] est l'envers de la quête. Sa doublure intime. Elle est ce que vous avez fui et conjuré, à force d'énergie et de combats, votre vie durant. [...] En termes aérologiques, j'appelle ça l'essoufflement. Les abrités sont avant toute autre critique des essoufflés.

VERCAUTEREN, David. Micropolitiques des groupes. Pour une écologie des pratiques collectives. Paris: Les Prairies ordinaires, 2011 [2007], p. 155:

En arabe ancien, Eilm exprime un savoir, une science singulière: celle des signes qui permettent aux nomades de se déplacer dans le désert sans se perdre. À ce savoir des signes correspond une manière d'envisager le trajet. Par habitude, nous concevons un trajet à partir de deux points, le départ et l'arrivée, mais pour un nomade il existe un troisième espace-temps qui a sa propre consistance et jouit d'une autonomie particulière: c'est l'entre-deux du trajet.

Voici quelques décennies qu'un contexte souvent dit « de crise " pousse à expérimenter des manières d'être, de faire et de connaître qui bousculent les pratiques et représentations instituées. Dans l'efflorescence des constellations qui s'activent ici et là pour cultiver, habiter, fêter, construire, résister, penser, lutter, etc. autrement, je voudrais m'intéresser au point de jonction et de transformation réciproque entre les institutions muséales et universitaires. Comment est-ce que l'on appelle " recherche » peut participer au renouvèlement des paradigmes obsolètes?

Du côté du monde de l'art, on s'entend pour considérer le processus de création, sinon l'œuvre, comme une manière singulière de savoir ${ }^{1}$. Les commissaires et artistes Aliocha Imhoff et Kantuta Quiros, par exemple, définissent le curating comme une série "d'opérations de connexion, de spatialisation, de montage, d'agencement, de traduction, que nous pourrions qualifier de pratiques de l'interstice ${ }^{2}$ ». Cette image de l'interstice, sur laquelle nous reviendrons plus précisément avec la première rencontre indisciplinaire, n'est pas sans parenté avec celle du delta mobilisée par Henk Slager dans un ouvrage qui revendique le plaisir d'une recherche artistique entendue comme l'invention d'une science située au croisement - au "delta »- des sciences dites " alpha » (Humanités), " beta » (sciences empiriques) et " gamma » (sciences impliquées) ${ }^{3}$. Bien que ce soit notamment par contraste et différenciation avec les institutions universitaires que se forgent ces

1 Dans un sens très large bien que non majoritaire, dans différentes formes esthétiques, organisations et manières de faire: pratiques de création coopératives ou dialogiques, centres d'art autogérés ou laboratoires, œuvres-enquêtes, etc. Voir, par exemple, le travail de franck leibovici (sic) ou de Till Roeskens.
2 IMHOFF Aliocha et QUIROS Kantuta. "Curating research - Pour une diplomatie entre les savoirs ", L'Art même $\mathrm{n}^{\circ} 64,2015$, p. 3-4.

3 SLAGER Henk. The Pleasure of Research, Ostfildern, Hatje Cantz, 2015, p. 76. 
nouvelles manières de faire, de penser et de créer, les universités aussi travaillent à renouveler leurs pratiques comme leurs conceptions de la recherche et de l'enseignement.

Du côté du monde universitaire, et plus particulièrement au Québec, le syntagme " recherche-création » désigne la volonté de prendre appui sur les œuvres et les processus créatifs pour renouveler les modes d'élaboration des savoirs - et renflouer une population étudiante en perte de vitesse ${ }^{4}$. En Europe, le processus de Bologne a suscité autant de réticences que d'analyses approfondies sur la différence et la complémentarité entre recherche et création, « recherche en art(s) vs. recherche artistique " (Yvane Chapuis), " recherche d'un non-savoir » (Yann Chateigné), " science de l'art » (lordanis Kerenidis et Théodora Domenach) - pour ne citer que quelques titres du seul numéro 130 de Culture et recherche paru à l'hiver 2014-2015'.

Le face-à-face réducteur entre " monde de l'art » (il faudrait, déjà, écrire " mondes des arts») et « monde universitaire » ne doit pas masquer l'importance des expérimentations qui ont lieu en dehors des institutions. Pour peu qu'on y prenne garde, on voit proliférer « en plein air ${ }^{6}$ " des recherches partenariales, coopératives, horizontales, en alliance et reliance, etc. Dans un ouvrage édité par un collectif significativement nommé « Les Chercheurs ignorants ", Yves Bonny esquisse une typologie distinguant la recherche collaborative, la recherche-action collaborative, la recherche-intervention et la recherche-action coopérative. Selon lui, seule cette dernière permet de " constituer un "acteur-chercheur hybride", dépassant les catégories de praticien et de chercheur, sans pour autant confondre les identités, les rôles et les contributions ${ }^{7}$ ».

Ma position, qui s'ancre dans l'université tout en s'inspirant des centres d'art pour travailler aussi en dehors des institutions, consiste à déployer une recherche que j'ai appelée « indisciplinaire ${ }^{8}$ ». L'enjeu est moins de remembrer les sciences spécialisées (comme le ferait l'approche interdisciplinaire) que de penser en amont de ces catégories, pour rebrancher savoirs, actions et créations dans une optique de transformation et d'émancipation. C'est dans cette perspective que

4 Voir par exemple: La recherche création. Pour une compréhension de la recherche en milieu artistique, (GOSSELIN Pierre et LE COGUIEC Éric dir.), Québec, Presses de l'Université du Québec, 2006. Au cours de l'année 20152016, Louis-Claude Paquin et Isabelle Caron ont mis en place à l'UQAM un incubateur d'idées autour des modalités de la recherche-création.

5 La recherche dans les écoles supérieures d'art, Culture et recherche $\mathrm{n}^{\circ} 130$, hiver 2014-2015, http://www.culturecommunication.gouv.fr/var/culture/storage/pub/culture_et_ recherche_130/index.htm

6 CALLON Michel, LASCOUMES Pierre et BARTHE Yannick. Agir dans un monde incertain. Essai sur la démocratie technique. Paris: Le Seuil, « La couleur des idées », 2001.
7 BONNY Yves. "Les recherches partenariales participatives: ce que chercher veut dire ", Les Chercheurs ignorants (dir.), Les recherches-actions collaboratives: Une révolution de la connaissance? Rennes: Presses de l'EHESP, 2015, p. 38. 8 SUCHET Myriam. Indiscipline! Tentatives d'UniverCité à l'usage des littégraphistes, artistechniciens et autres philopraticiens. Montréal: Nota Bene, "Indiscipline », 2016. Je me suis inspirée notamment de LOTY Laurent. « Pour l'indisciplinarité ». Dans DOUTHWAITE Julia et VIDAL Mary (éd.). The Interdisciplinary Century. Oxford: Studies on Voltaire and the Eighteenth Century, 2005, p. 245-259. Et aussi de CITTON Yves. Lire, interpréter, actualiser. Pourquoi les études littéraires? Paris: Éditions Amsterdam, 2007. 
j'ai mis à profit mon séjour à Montréal en tant que titulaire de la Chaire d'études de la France contemporaine, entre janvier et juin 2015. II ne s'agissait pas de venir énoncer au Québec un savoir déjà constitué à propos de la France contemporaine, mais plutôt de générer des frottements pour voir ce qui en résulterait en matière de rechercheaction-création. Pour ce faire, j'ai mis en place un cycle de cinq " rencontres indisciplinaires ", chacune imaginée en complicité avec une personne invitée dans un lieu différent et un format spécifique (en évitant les dispositifs attendus du type colloque, journée d'étude, conférence, table dite "ronde » ou symposium) autour d'un objetnavette favorisant la mise en relation entre les mondes de la recherche, des arts et de la pratique - chacun d'entre eux étant rarement un seul à la fois.

Une telle recherche relationnelle ne peut s'élaborer que chemin faisant, au gré de rencontres effectives. D'où l'importance des retours sur expérience, qui permettent de formuler et de formaliser - jusqu'à la prochaine transformation - les étapes d'un devenir. D'où la difficulté, aussi, de trouver une forme appropriée: tandis que l'article scientifique sied mal à une pensée en chantier perpétuel, le journal est trop intime pour une démarche aussi collective. C'est avec Pierre Tandille que j'ai d'abord essayé de traduire graphiquement - et, ce faisant, de continuer à mettre en forme - un programme où les rencontres s'agençaient comme des flux, des champs de forces, avec des points de cristallisation et des lieux de passage privilégié. L'idée du vent nous est venue à partir d'une référence commune: La Horde du Contrevent d'Alain Damasio. En m'offrant la forme du "carnet », l'équipe Arts des mondes/ Mondes des arts témoigne de sa générosité et me met au défi de " transformer l'essai » de notre rencontre avec Dominic Hardy et l'équipe du DHC/ART. Puisse cette tentative être à la hauteur de leur accueil et de notre travail commun!

La première rencontre: ouvrir le champ des possibles

\section{Point d'inspiration}

NICOLAS-LE STRAT, Pascal. « Multiplicité interstitielle», Multitudes 4, n 31, 2007, p. 115-121. URL: www.cairn.info/ revuemultitudes-2007-4-page-115.htm (consulté le 1"r avril 2017)

Les interstices représentent ce qui résiste encore dans les métropoles, ce qui résiste aux emprises réglementaires et à l'homogénéisation. Ils constituent en quelque sorte la réserve de " disponibilité » de la ville. Du fait de leur statut provisoire et incertain, les interstices laissent deviner ou entrevoir un autre processus de fabrication de la ville, 


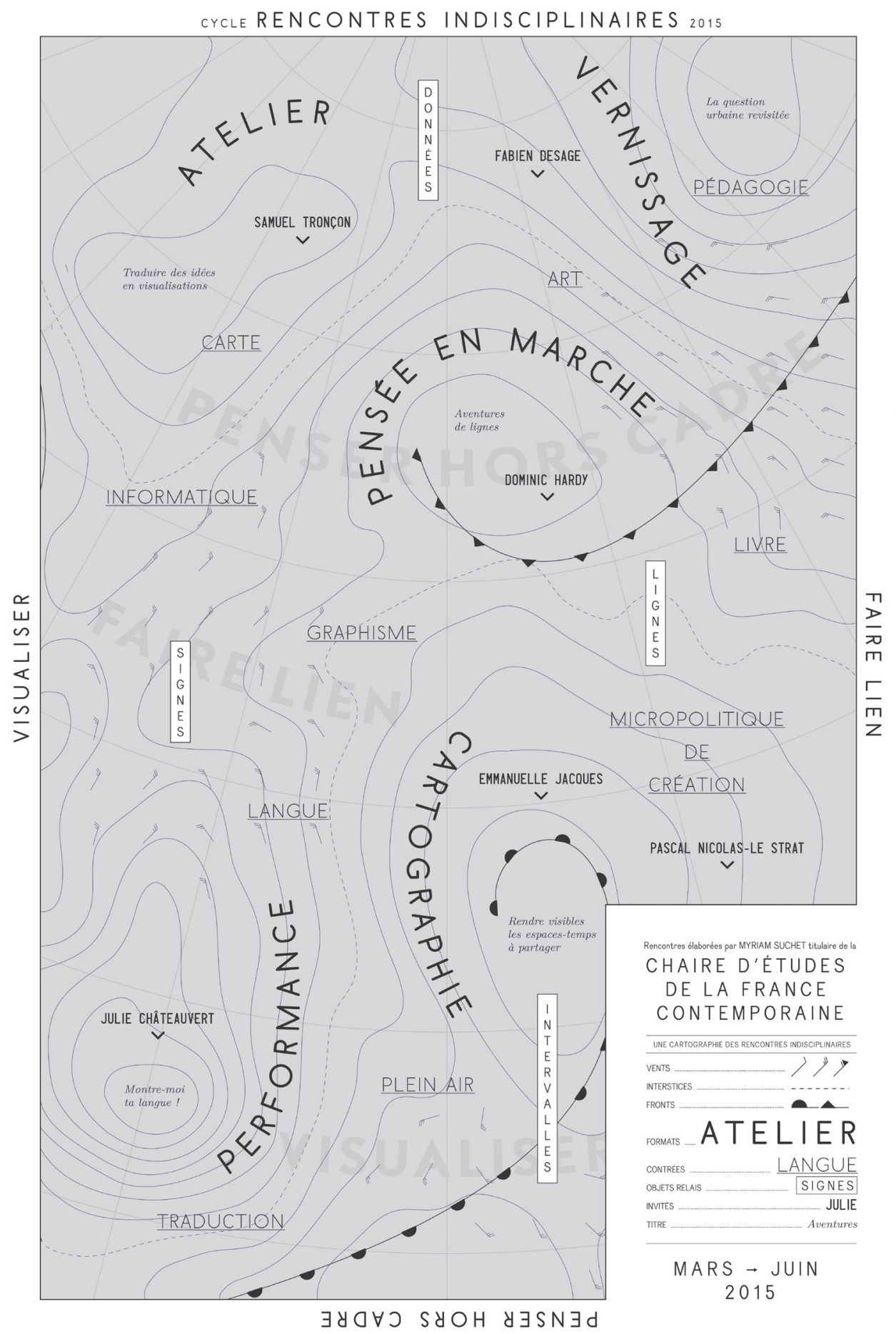

Fig. 1 
ouvert et collaboratif, réactif et transversal. C'est effectivement sur ce plan-là, à la fois méthodologique et formatif, politique et heuristique, que se vérifie l'importance de l'expérience interstitielle.

\section{Dispositif}

Date: Vendredi 13 mars 2015

Titre: Intervalles $\cap$ interstices $\cap$ battements

Complices:

- PASCAL NICOLAS-LE STRAT, Sociologue du travail, Professeur à Paris 8

- et EMMANUELLE JACQUES, Artiste visuelle et cartographe Invité.e.s:

- SIMON HAREL, Professeur et Directeur du Département de littératures comparées à I'UdeM, directeur du Laboratoire des récits du soi mobile

- LUC LÉVESQUE, Professeur à I'Université Laval, cofondateur de SYN (atelier d'explorations urbaines)

- ROGER LATOUR, Auteur et Naturaliste urbain, Président fondateur du projet Les Amis du Champ des Possibles

- GENTIANE BÉLANGER, Doctorante en histoire de l'art à I'UQAM

Lieu: Studio XX, 4001, rue Berri, Montréal H2L 4H2

Format: Rencontre et atelier cartographique

\section{Ressaisie}

La toute première rencontre s'est organisée autour de l'invitation du sociologue Pascal Nicolas-Le Strat. Après quelques tâtonnements et discussions avec Gentiane Bélanger du côté de l'histoire de l'art et Simon Harel en littérature comparée, nous avons mis en place un atelier cartographique avec l'artiste visuelle Emmanuelle Jacques. L'objectif était d'articuler la réflexion de Pascal Nicolas-Le Strat sur les interstices urbains avec une pratique concrète de l'espace montréalais et, plus spécifiquement, du Champ des possibles. En articulant pratique et théorie, il s'agissait aussi d'actualiser son idée du commun ${ }^{10}$ pour produire de nouvelles connaissances mutualisées.

L'atelier, qui s'est tenu au Studio XX, s'ouvrait sur un buffet préparé par l'écrivain Michel Vézina. Les chaises étaient disposées en cercle, les tables couvertes de nappes de papier, de tampons encreurs et de feutres de toutes les couleurs. Pour présenter son travail sans verser dans la conférence, Pascal Nicolas-Le Strat opte pour la forme d'un récit, d'une " scientifiction " comme il l'appellera ultérieurement dans son Journal ${ }^{11}$. L'articulation avec les autres interventions renforce cette inscription concrète du théorique: nous sommes convié.e.s à matéria-

10 NICOLAS-LE STRAT Pascal. Le Travail du commun. Rennes: Éditions du commun, 2016.
11 NICOLAS-LE STRAT Pascal. Journal (mars 2015 / février 2016). Chronique de l'activité d'un enseignant-chercheur, maquette sous Scribus: Benjamin Roux, imprimé par les Éditions du Commun en format A4 mais non diffusé, ce qui constitue aussi une expérimentation de forme de publication. 
liser notre compréhension de la logique interstitielle.

De cette première rencontre, je retiendrai l'importance du facteur temps. D'abord parce que les interstices s'avèrent relever autant d'une dimension temporelle que spatiale. Ensuite parce qu'il m'apparaît rétrospectivement à quel point $c^{\prime}$ est dans la longue durée que s'est tramée cette rencontre. Il y aura eu, d'abord, la découverte éblouissante d'un article de Pascal Nicolas-Le Strat, il y a plusieurs années déjà ${ }^{12}$. Puis mon implication croissante aux Fabriques de sociologie à Saint-Denis ${ }^{13}$, de multiples échanges à distance avec les invité.e.s, une expédition dans la neige avec Emmanuelle Jacques au Champ des possibles... En amont de l'atelier au Studio XX, nous avions aussi organisé deux temps de travail à l'Université de Montréal, l'un réservé au séminaire d'épistémologie littéraire, l'autre partagé avec les étudiant.e.s du Cégep de Saint-Jean-surRichelieu et leurs professeurs Frédéric Legris et Jonathan Naud. Ensemble, nous avons réalisé une édition-fresque collective de son article "Des compétences indisciplinées ». La réussite d'une rencontre, et j'en discute le soir même avec le collectif indisciplinaire Doyon/Demers, tient autant à son instant précis qu'à une expérience accumulée, une complicité longuement préparée.

\section{Aperçu}

Les photographies (sauf celle de l'éditionfresque) ont été prises par Maia lotzova, l'une des membres fondatrices du Wild City Mapping qui tenait un atelier au même Studio XX la semaine précédent notre rencontre: jonction établie!

Voir aussi la vidéo « À la découverte des interstices urbains » réalisée par Yasmine Berthou au Consulat général de France à Québec: https://www.youtube.com/ watch? $v=e 6 \mathrm{Ci} 6 \mathrm{Gi} 4 \mathrm{HKI}$

\section{Prolongations}

12 À l'occasion d'un colloque organisé par Sherry Simon et qui a donné lieu à une publication à quatre mains: SUCHET Myriam et MEKDJIAN Sarah. "Artivism as a Form of Urban Translation: An Indisciplinary Hypothesis ». Dans Speaking Memory, (SIMON Sherry dir.), Montréal: McGill Queen University Press, p. 220-248.

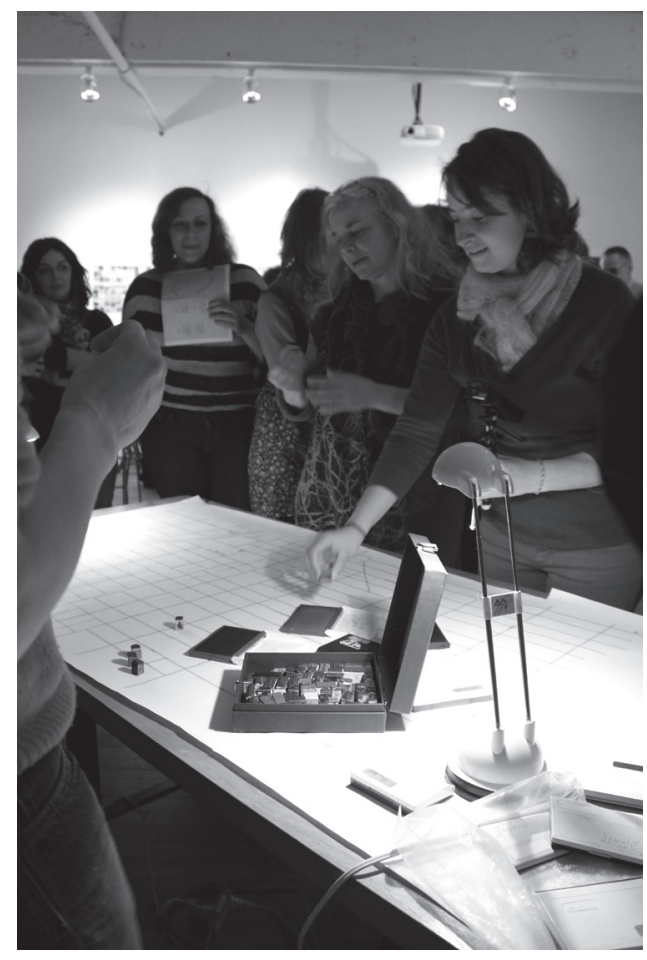

Fig. 2

Photographie de la rencontre $\mathrm{n}^{\circ} \mathbf{1}$, par Maia Iotzova
13 Collectif créé par Pascal Nicolas-Le Strat et Martine Bodineau, qui se présente comme " un espace de recherche en sciences sociales qui n'est pas réservé aux seuls chercheur-euse-s mais qui associe des professionnels de plusieurs champs d'activité (art, social, éducation, urbain, architecture), des militants et activistes, des autodidactes de la recherche, des étudiant-e-s en sciences sociales, des chercheurs universitaires ou non ", cf. http://www.fabriquesdesociologie.net/ 
Cette rencontre aura contribué à renforcer et à dynamiser des recherches des deux bords de l'Atlantique. Côté français, Pascal-Nicolas Le Strat et moi-même avons mis en place un collectif dans le cadre du Campus Condorcet (2016-2018): Fabriquer la ville "en-commun ». Des collectifs $d$ 'artistes et/ou d'architectes en recherche de nouvelles écritures urbaines. Côté montréalais, je prendrai part au « Catalyseur d'Imaginaires Urbains », infrastructure de recherche-création mise en place par Simon Harel sur le futur campus MIL de I'Université de Montréal (http:// leviragemtl.com/fr/).

Surtout, cette première rencontre sera prolongée par une carte-parcours-de-lecture réalisée par Emmanuelle Jacques (et mise en page par Pierre Tandille) qui associe - tentative inédite dans son œuvre - du texte aux étampes. Sa technique visuelle devient un mode exploratoire pour parcourir un article scientifique, s'y repérer et l'augmenter d'un terrain. La légende, que nous avons élaborée ensemble, donne des balises que vous pouvez à votre tour suivre ou déplacer en parcourant ce champ des possibles de lecture. Au dos de cette carte, nous éditons une version remaniée de l'article « Multiplicité interstitielle » de Pascal Nicolas-Le Strat:

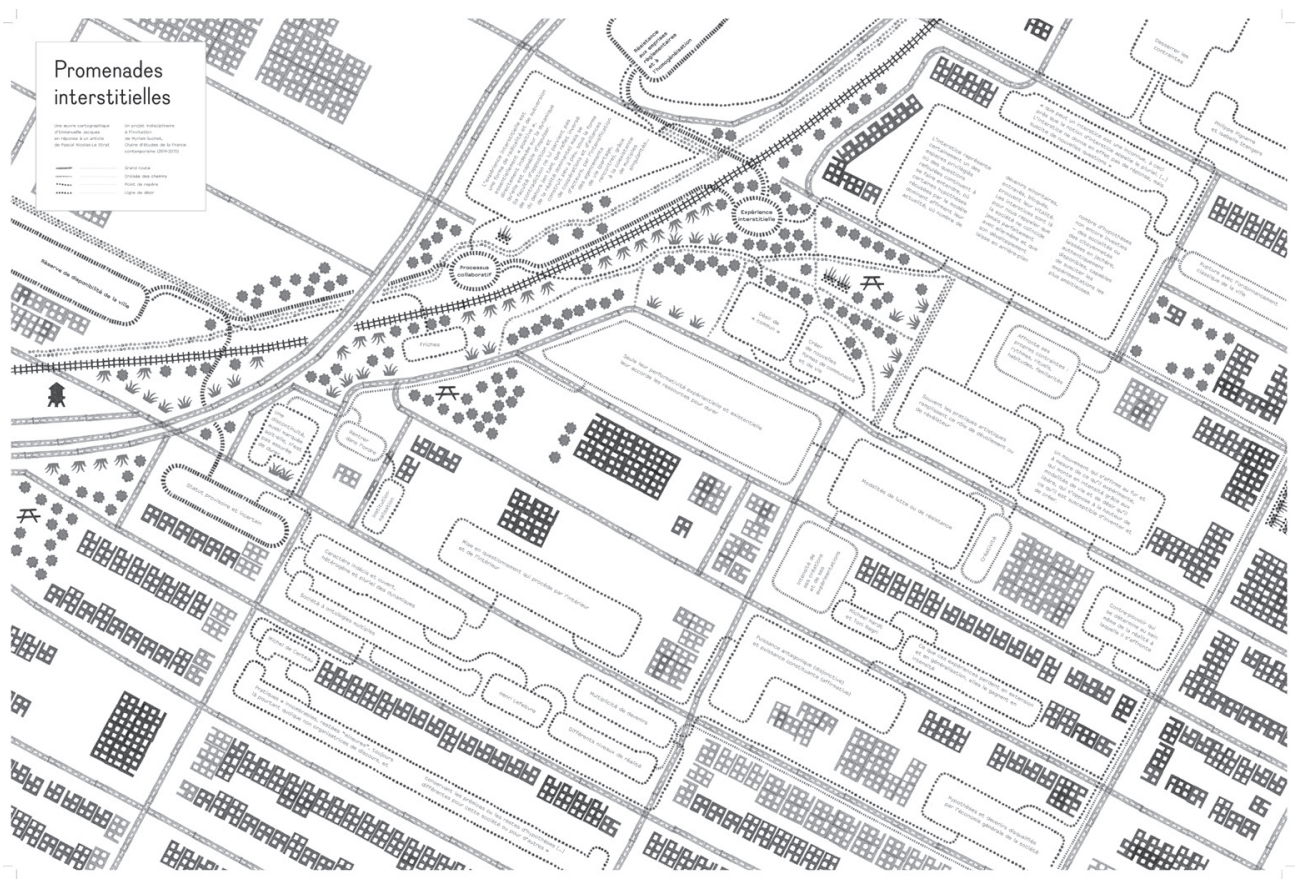

Fig. 3

La carte-parcours de l'article « Multiplicité interstitielle » par Emmanuelle Jacques 
Rencontre 2: on peut voir ce que vous faites?

\section{Point d'inspiration}

MICHAUX Henri. « Peindre (1938) », Passages. Paris: Gallimard, 2001 [1950], p. 322-323.

Le déplacement des activités créatrices est un des plus étranges voyages en soi qu'on puisse faire. Étrange décongestion, mise en sommeil d'une partie de la tête, la parlante, l'écrivante (partie, non, système de connexion plutôt).

On change de gare de triage quand on se met à peindre.

La fabrique à mots (mots-pensées, mots-images, mots-émotions, mots-motricité) disparaît, se noie vertigineusement et si simplement.

[...] C'est une expérience surprenante.

Et quel repos!

\section{Dispositif}

Date: vendredi 24 avril 2015

Titre: Donner à voir une pensée qui (se) cherche Invité: Samuel Tronçon, directeur de l'Agence de recherche-action en innovation sociale et visualisation de données Résurgences (Marseille - www.resurgences.eu)

Lieu: Laboratoire Hexagram, Université Concordia, Pavillon EV, 1515, Sainte-Catherine Ouest, Montréal, H3G 2W1

Format: atelier de visualisation de données

\section{Ressaisie}

L'enjeu de cette rencontre, orchestrée avec Samuel Tronçon, était de donner accès à la boîte noire d'une recherche en cours. Graphes, cartes heuristiques, et autres arborescences: les outils mis en œuvre par l'agence Résurgences semblent permettre à la fois d'exposer les résultats d'un travail et de donner à voir son mode opératoire.

La rencontre s'est déroulée en deux temps: une journée d'atelier offerte aux étudiants et aux étudiantes de doctorat du laboratoire de recherche-création Hexagram (imaginée avec Natalie Lafortune), puis une soirée de restitution ouverte au grand public. L'idée était à la fois d'offrir un temps d'expérimentation aux jeunes chercheurs et chercheuses (travail de synthèse, maniement des codes visuels, délinéarisation de la réflexion...) et d'éprouver avec eux une hypothèse: passer d'une organisation discursive à une forme graphique peut faire émerger des zones et révéler des rapports qui seraient sinon restés inaperçus. 
L'apport le plus manifeste de ce travail - en laissant de côté la sensibilité esthétique de chaque personne - aura été la mise en exergue des questions logiques: I'organisation des hypothèses et des corpus, l'orchestration des données et la hiérarchie des étapes du raisonnement ont été questionnées, remaniées, explicitées. De cette interrogation logique a découlé une réflexion sur la pertinence, ravivant la citation de René Thom: "Ce qui limite le vrai, ce n'est pas le faux, c'est l'insignifiant ${ }^{14} »$.

Une autre conséquence est celle du passage d'un mode individuel à une élaboration collective de la recherche. Chaque personne reste " spécialiste " de son sujet, mais toutes ont participé avec des apports complémentaires à tel ou tel aspect non seulement de sa présentation, mais aussi de sa conception.

\section{Aperçu}

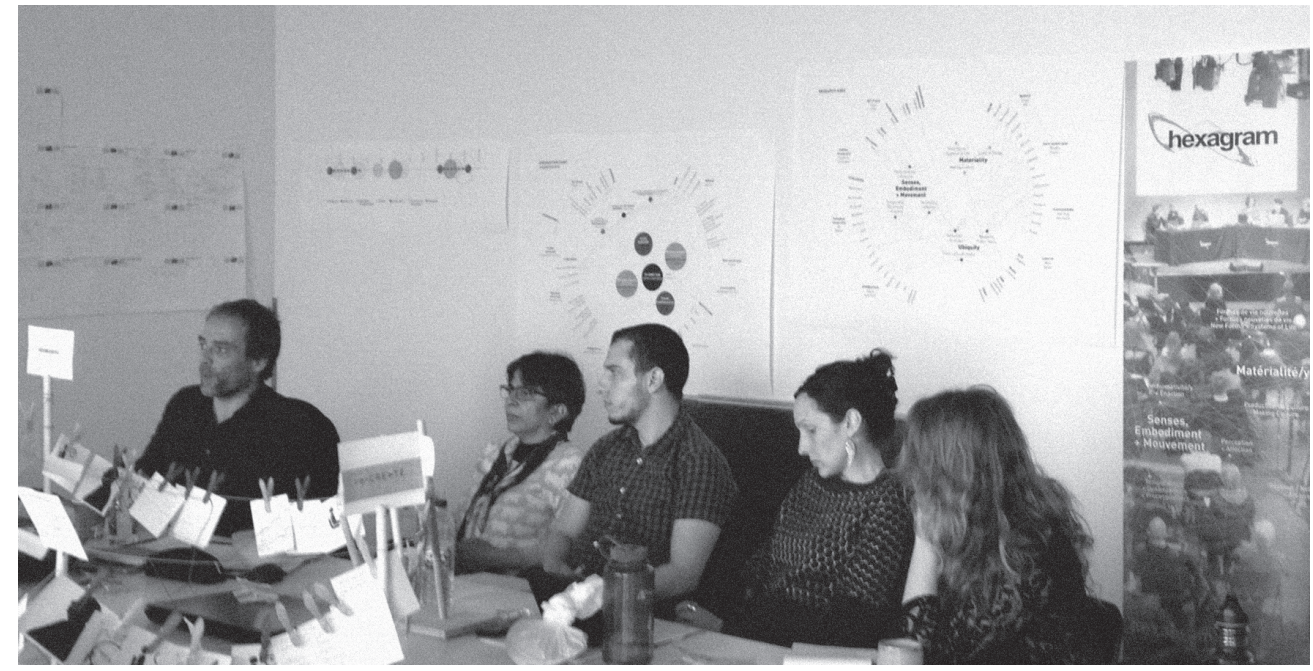

Fig. 4

Premier temps de la rencontre avec Samuel Tronçon: l'atelier Hexagram

\section{Prolongation}

Dans la prolongation de cette rencontre, deux autres ateliers ont été offerts aux doctorants et aux doctorantes d'Hexagram. Le premier, La pensée graphique: réussir un poster ou une carte heuristique d'un point de vue de la forme, de la taille et de la couleur, nous a de nouveau conduit au Studio XX (le 7 juin 2015). La graphiste Jessica Charbonneau ${ }^{15}$ est intervenue avec des compétences complémentaires

14 THOM René. Prédire n'est pas expliquer. Paris: Eshel, 1991, p. 132.
15 Voir www.studiotagteam.com. J'ai rencontré Jessica Charbonneau grâce à Céline Poisson, Professeure à l'UQAM, avec qui elle travaille notamment les "Espaces de savoir " publiés aux Presses de l’Université Laval. 
à celles de Samuel Tronçon. Le second, Intervenir brièvement devant un poster: retour du visuel au discursif (6 octobre 2015), consistait à travailler la prise de parole nécessaire pour accompagner une visualisation de recherche. Nous avons travaillé avec Ha-Loan Phan, coordinatrice de communication scientifique à l'ACFAS, Hanen Allouch, doctorante à l'Université de Montréal en littérature comparée (qui suivait le séminaire d'épistémologie littéraire) et Cynthia Noury, coordonnatrice à la recherche pour Hexagram ${ }^{16}$.

Par ailleurs, nous avons profité de la présence de Samuel Tronçon pour participer aux journées d'étude Ambiances en traduction (27-29 avril 2015 à l'Agora Hydro-Québec) $)^{17}$ avec le collectif de "Traduction indisciplinaire » que j'ai composé avec Catherine Leclerc (McGill), Hélène Buzelin (UdeM), Sherry Simon (Concordia), Raúl Colón (Ottawa), Julie Châteauvert (UQAM) ainsi que deux traductrices militantes: Anna Sheftel et Patricia Boushel, instigatrices des plateformes «Translating the Printemps Erable ${ }^{18}$ » et « Language and Dissent ${ }^{19}$ ».

Last but not least, l'Agence Résurgence a développé un outil de travail coopératif en s'inspirant de l'atelier Hexagram: Insight, auquel nous contribuons par trois groupes de recherche: le projet de pédagogie innovante Agilabil, le groupe de travail Art et création comme résistance et la publication du numéro Traduire de la revue Intermédialités.

Rencontre 3: là, je donne ma langue au chat

\section{Point d'inspiration}

\section{Julie Châteauvert}

On traduit rarement d'une langue des signes vers une langue écrite. Pour diverses raisons. Le reflet d'une organisation du monde. Le politique se manifeste notamment dans cette asymétrie. Décider $d^{\prime} y$ toucher est donc une manœuvre délicate. Il y aurait d'abord tant à créer. S'en approcher toutefois permet de découvrir toute l'ingéniosité des œuvres et nous entraîne dans un parcours réflexif à propos de la traduction jalonné d'étonnements. C'est qu'il s'agit d'œuvres intermédiales qui se jouent des stratégies de traduction communes. Comment transposer en français une suspension du mouvement? Comment traduire l'instant qui fait surgir dans l'imaginaire une fresque de la renaissance?

16 Voir http://www.hexagram.ca/index.php/fr/ blog-evenements-passes/26-workshop/170-atelier-7 17 Voir http://www.ambiances.net/seminars/gdri-translating-ambiances-montreal2015.html
18 Voir http://translatingtheprintempserable.tumblr.com/

19 Voir https://languageanddissent.com/ 


\section{Dispositif}

Date: Samedi 25 avril 2015

Titre: Montre-moi ta langue!

Invités: Julie Châteauvert et Daz Saunders, ainsi que Valérie Lefebvre-

Faucher, Véronique Bachand, Pamela Witcher, Joel Lemay, Véronique

Leduc, Courtney Lester

Lieu: Cerium (bureau 530-1), Université de Montréal, 3744, Jean-Brillant

Format: Atelier hétérolingue de traduction en langues signées

(sur invitation)

\section{Ressaisie}

Pour cette rencontre, je me suis laissée guider hors de mon périmètre de compétence et même de compréhension par Julie Châteauvert et Daz Saunders, complices de longue date dans leur exploration des créations en langues des signes ${ }^{20}$. $\mathrm{J}^{\prime}$ insiste sur le pluriel ici, qui était la cheville ouvrière de notre expérimentation: il s'agissait d'orchestrer, autour d'une traduction poétique, plusieurs langues signées: la Langue des Signes du Québec (LSQ), la Langue des Signes Française (LSF), l'American Sign Language (ASL) et la British Sign Language (BSL).

Chacune des interventions était traduite dans l'une des langues des signes en présence: $A S L, B S L, L S F$ et LSO ainsi qu'en français parlés (au pluriel). Les œuvres poétiques de Debbie Rennie et du Flying Words Project, à partir desquelles nous avons travaillé, peuvent être visionnées ici : https://ecoleduregard.wordpress.com/les-oeuvres-etudiees/.

Inversement, le groupe a traduit une strophe d'Henri Meschonnic en LSQ, en LSF et en ASL. La fin a particulièrement suscité la discussion: " quand je me réveille de mes moments sourds [...] je ne connais pas ma main ».

Les langues des signes $m$ 'intéressent à plusieurs titres, bien que je n'en maîtrise aucune. Outre la question des rapports de force, qui n'est pas sans écho avec les différentes situations postcoloniales, les possibilités énonciatives signées interrogent la conception du sujet parlant. Les langues signées permettent en effet de se mettre en scène en tant qu'autre, modalité passionnante de diffraction ou de ventriloquie - davantage que de simple délégation - énonciative. Pour citer des propos tenus par un ou une autre, la personne qui signe endosse son rôle: "C'est ce qu'on appelle aussi souvent la prise de rôle, avec actant, procès, locatif. Le locuteur devient l'entité dont il parle. Tout le corps du locuteur est occupé par le rôle (pas de recul, contrairement au cas du transfert de situation): il y a incorporation ${ }^{21}$.

20 Voir http://www.upopmontreal.com/hiver-2015/686-2/

21 SALLANDRE Marie-Anne. "Va-et-vient de l'iconicité

en langue des signes française ", Acquisition et interaction en

langue étrangère, $\mathrm{n}^{\circ} 15,2001$, http://aile.revues.org/1405

(consulté le 29 janvier 2017). 
Cette possibilité énonciative devient « technique (ou conviendrait-il mieux de dire figure?) lorsqu'elle est le pivot essentiel d'une performance narrative 122 comme dans le cas du Wise Old Corn analysé par Julie Châteauvert et expérimenté dans notre atelier. L'entrée esthétique, loin d'oblitérer ces enjeux et les luttes pour la reconnaissance et l'émancipation, permet de les envisager dans une complexité salutaire et de les outiller autrement. Éprouver la différence des poétiques signées et l'éventail des possibles traductionnels dans ces mêmes langues, c'est consolider un répertoire artistique et théorique $c^{\prime}$ est aussi prendre acte d'une puissante capacité à dire/signer.

Notre cercle de travail s'est penché sur l'hétérogénéité de "la langue " - aux antipodes de l'idée reçue selon laquelle il existerait une langue des signes universelle ou que les différentes langues signées ne constitueraient pas des langues à part entière. Cette question était en quelque sorte déjà dépassée. Nous étions à un autre plan ou stade de réflexion, qui nous conduisait dans l'atelier où les artistes éprouvent les formes, les essayent, les peaufinent. En fait, la langue parlée n'était plus un étalon ni un point de référence: les jeux que permettaient les signes et leur organisation se faisaient indépendamment du français ou de l'anglais parlé. Nous éprouvions (y compris l'entendante que je suis) la puissance de frayage de l'énonciation signée: l'iconicité et la syntaxe codée des langues des signes permettent, comme dans toute langue, des effets de style qui continuent à repousser l'horizon des possibles - et à exiger des méthodes et des outils d'analyse spécifiques.

\section{Prolongation}

Recentré autour de l'hétérolinguisme à mon retour en France, Montre-moi ta langue! (titre dont l'impertinence ne fonctionne ni en LSF ni en LSQ), devient un programme du Centre d'Etudes Québécoises de la Sorbonne Nouvelle - Paris 3. Il s'agit d'éprouver le potentiel de l'imaginaire hétérolingue pour connecter le mode de réflexion caractéristique de l'université avec les pratiques expérimentales menées hors de l'institution universitaire. Parmi les événements organisés a eu lieu notamment la première édition de la Pensée coulisse intitulée "Danses des signes et autres jeux de mots \& mains » au Le Tarmac, Scène internationale francophone (Paris, le 15 décembre 2016). Julie Châteauvert a participé à cette rencontre, imaginée autour du spectacle Suites curieuses avec la Compagnie Cas public, Julien Bourguignon (responsable des publics), Hélène Blackburn (chorégraphe), Bachir Saïfi (comédien), Isabelle Florido, (comédienne), Nicolas Fagart (metteur en scène), Isabelle Lombard et Vincent Bexiga (interprètes LSF/français). 


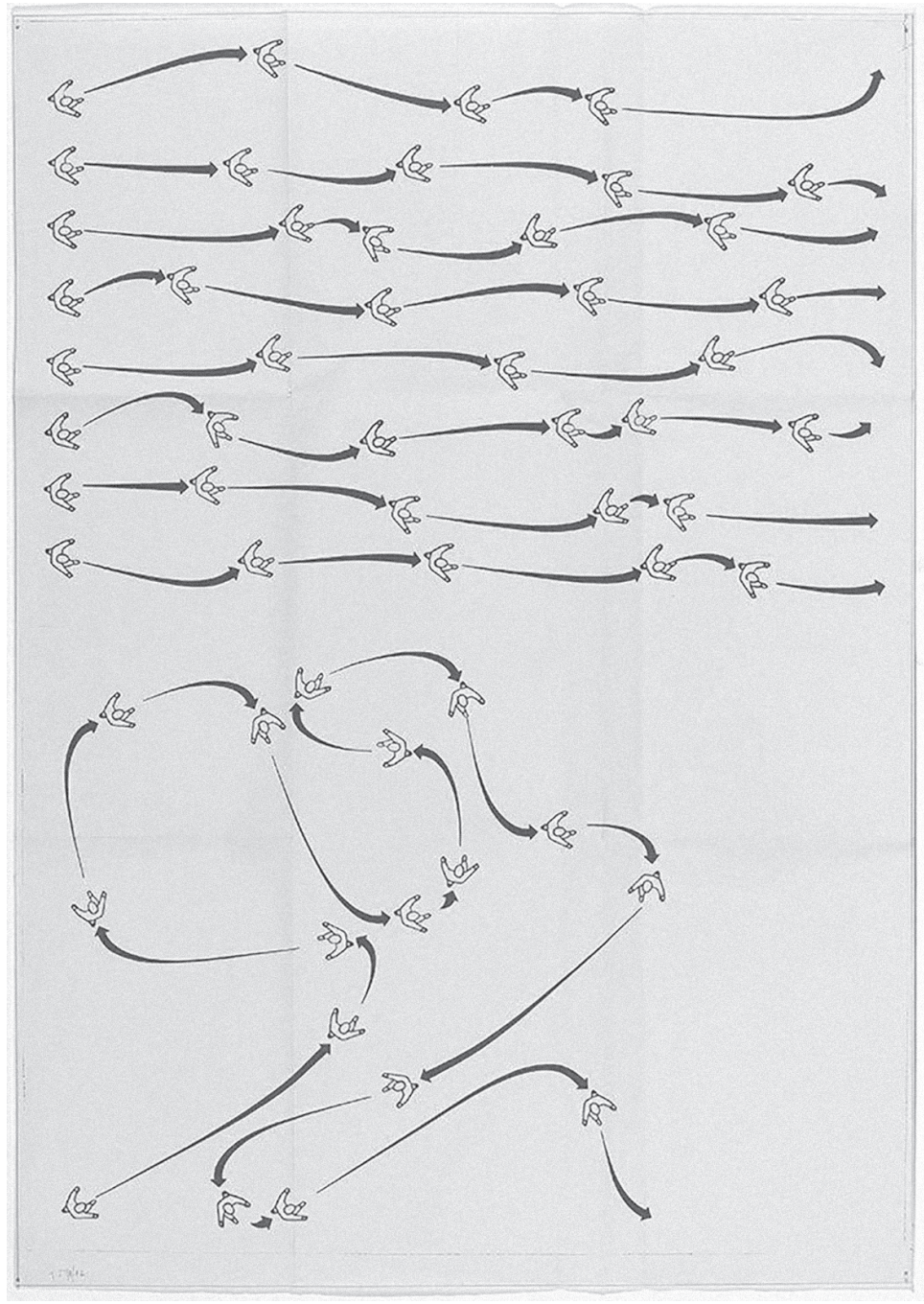

Fig. 5

Léon Ferrari, Caminos 


\section{Rencontre 4, Aventures de lignes}

\section{Point d'inspiration}

FERRARI Léon, Caminos, 1982 - exposé au centre Pompidou Metz en écho à INGOLD Tim. Une brève histoire des lignes, trad. Sophie

Renaud. Bruxelles: Zones Sensibles, 2011 [2007]

\section{Dispositif}

Date: Lundi 25 mai 2015

Titre: Aventures de lignes

Invité: Dominic Hardy (UQAM), en complicité avec les étudiant.e.s Giuditta Lorenzini et Daniel Boisclair ainsi que l'équipe de médiation du DHC/ART: Emily Keenlyside, Amanda Beattie, Daniel Fiset et Marie-Hélène Lemaire Lieu: DHC/ART \& alentours, 451, rue Saint-Jean, Montréal Formats:

- Visite déguidée du Vieux-Montréal: port et ancrages

- Pensée en marche et en mouvement au DHC/ART

- Atelier de création: traces en surimpressions

\section{Ressaisie}

Cette quatrième rencontre indisciplinaire s'est articulée autour de I'exposition Pièces de résistance de Yinka Shonibare MBE. L'une des marques de fabrique de l'artiste est le tissu wax dont il habille l'univers du $18^{e}$ siècle et de l'ère victorienne qu'il revisite dans ses toiles et ses sculptures. Dans son œuvre stratifiée, le wax raconte une histoire connectée, une succession d'ondes de choc et de résonances d'Indonésie en Europe, en passant par l'Afrique de l'Ouest. Yinka Shonibare invite à interroger la manière dont l'art se saisit des nœuds qui tissent nos contemporanéités.

En complicité avec l'équipe de médiation du DHC/ART, nous avons combiné trois dispositifs: une visite déguidée du Vieux-Port de Montréal pour interroger l'ancrage spatial, historique et imaginaire de cette exposition, une " pensée en marche " dans l'exposition pour faire l'expérience d'un collectif interprétatif émergeant de la rencontre avec l'œuvre et enfin un atelier de création pour mettre la main à la pâte et composer, ensemble, une réponse au travail de Shonibare.

La « visite déguidée » du Vieux-Montréal connectait l'espace de l'exposition à celui de la ville pour renouer le fil de récits fictifs et bien réels d'une histoire traumatique. Elle a été orchestrée par Dominic Hardy ainsi que Giuditta Lorenzini et Daniel Boisclair, au doctorat à I'UdeM. À l'issue de cette mise en jambe, nous avons activé une «pensée en marche ». Ce format de visite (que j'ai mis en place en 
2011 au macLYON ${ }^{23}$ ), consiste à faire intervenir des chercheuses et des chercheurs venus de différentes disciplines dans les espaces d'exposition du musée, en présence d'une personne médiatrice qui facilite les échanges avec le public. Invitée au musée, la pensée universitaire ne disserte plus sur un objet d'étude mais avec lui. Chaque intervention frotte les concepts aux œuvres, déstabilisant aussi bien la spéculation des chercheurs que l'horizon d'attente des visiteurs. Cette déstabilisation réciproque nourrit des échanges à la suite de chaque intervention, limitée à une durée maximale de 10 minutes. Une réflexion d'ensemble se construit chemin faisant, en passant d'œuvre en œuvre et d'intervention en débat. Le verbe " marcher " s'entend donc dans un double sens: il s'agit, d'une part, d'arpenter les salles de l'exposition et, d'autre part, de faire fonctionner la pensée. Pour le musée, il s'agit d'offrir un mode de visite inédit, qui prend appui sur le travail des artistes et qui l'articule aux avancées de la recherche pour susciter une réflexion critique. Dans l'expérience du DHC/Art, cette "pensée en marche " s'est imaginée directement avec l'équipe du musée et a débouché sur un atelier de pratiques qui correspond à leurs activités pédagogiques et inspirantes.

\section{Aperçu}

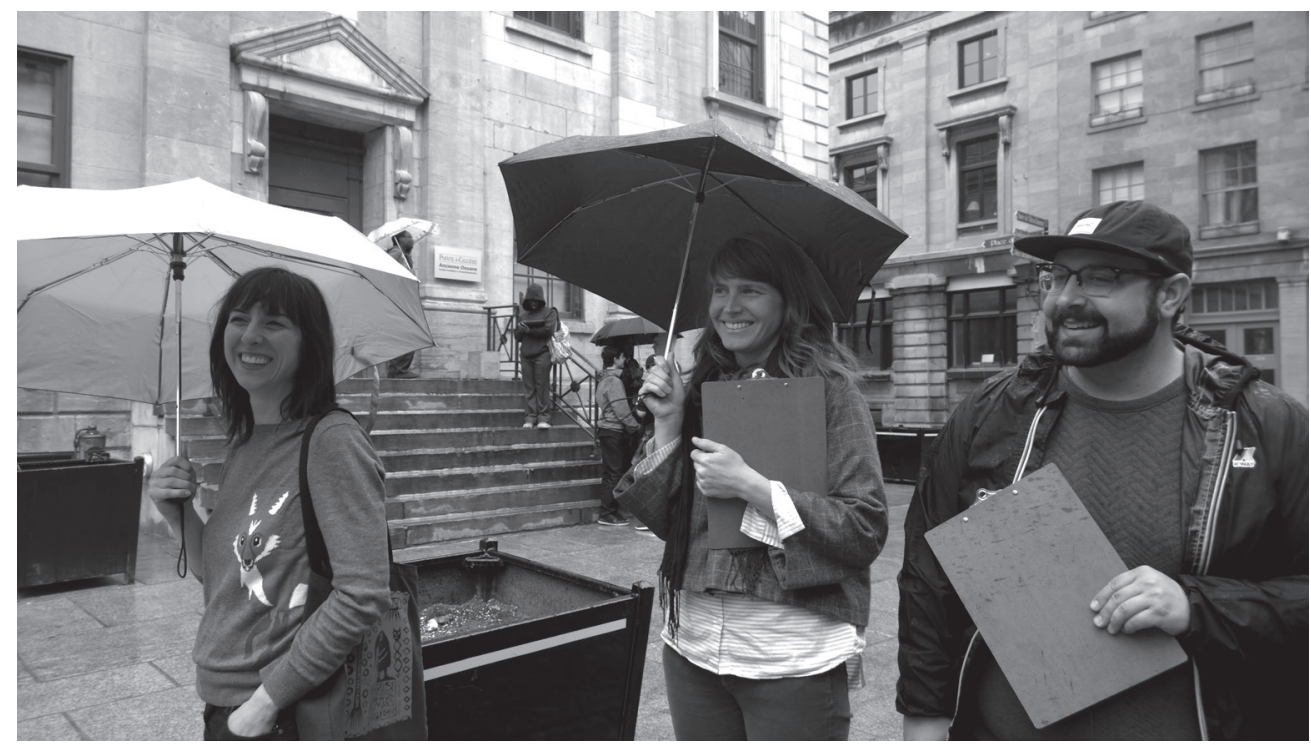

Fig. 6

Photographie de la rencontre (visite déguidée, pensée en marche et atelier) au DHC-ART 


\section{Prolongation}

La rédaction de ce " carnet " constitue en elle-même un rebondissement à notre rencontre. C'est aussi une réponse à l'article coécrit par Amanda Beattie, Daniel Fiset, Emily Keenlyside et Marie-Hélène Lemaire, réuni.e.s avec l'artiste-chercheure-pédagogue Pohanna Pyne Feinberg à l'occasion du dixième anniversaire du $\mathrm{DHC} / \mathrm{Art}$. Ce texte, qui explicite les principes de leur approche éducative, intègre à leur réflexion au long cours le moment de notre "Pensée en marche » et discute de sa proposition « indisciplinaire ».

Notre rencontre a aussi rebondi de l'autre bord de l'Atlantique, lors de deux interventions dans des centres d'art: à l'occasion de la résidence de création et de recherche orchestrée par Lotte Arndt à la Villa Vassilief dans le cadre du Goethe Institut Fellowship (" Pour parler de ma demeure, il me faut aussi parler de la demeure de l'autre ", samedi 10 décembre 2016) et lors des journées d'étude « Pratiques de co-création » organisées au MacVal et au Cac Bretigny par Céline Poulin, Marie Preston et Stéphanie Airaud.

\section{Rencontre 5, indisciplines croisées}

\section{Point d'inspiration}

DESAGE Fabien. «"Enfantillages" : photographier les enfants (et leurs parents) dans l'espace public ». Metropolitiques, 2015, http://www.metropolitiques.eu/Enfantillages-photographier-les.html

Je suis sociologue du politique avant d'être photographe. Pourtant, à force de multiplier les clichés et les projets ces dernières années, de parcourir avec avidité les monographies consacrées à quelques " grands photographes » ou encore de baguenauder sur les innombrables sites ou blogs dédiés à la photographie, j'ai fini par me convaincre qu'il ne s'agissait pas seulement d'un "loisir ", pratiqué en dilettante, mais que cette activité participait de mon identité de chercheur en sciences sociales et de mon intérêt pour les questions urbaines notamment; et, par extension, que le résultat de ce travail méritait peut-être d'être montré, soumis aux regards et aux jugements extérieurs.

\section{Dispositif}

Date: Vendredi 5 juin 2015

Titre: La question urbaine revisitée: Enfantillages Invité: Fabien Desage, sociologue et photographe, et Marie-Pierre Labrie, éducatrice artistique et artiste, membre du Centre de création pédagogique Turbine Lieu: Café Le Fixe, 5985, rue Saint-Hubert, Montréal

Format: vernissage d'exposition 


\section{Ressaisie}

Cette dernière rencontre s'est imaginée en complicité avec Fabien Desage, sociologue de la politique (à I'Université de Lille, France) et titulaire de la Chaire d'études de la France contemporaine avant moi. La piste indisciplinaire a révélé un pan masqué de son travail: la photographie, qu'il pratique en amateur mais aussi en chercheur, s'intéressant notamment à la manière dont elle peut être mobilisée dans le cadre des sciences sociales et des pratiques militantes. Nous avons convenu d'exposer la série de ses photographies d'enfants dans la ville, de sorte à interroger à la fois l'indiscipline de l'enfantillage et celle de sa démarche.

L'exposition, présentée au café Le Fixe, a aussi servi de cadre à un dialogue avec une autre indisciplinée, Marie-Pierre Labrie, dont le travail se situe à la croisée de la pratique artistique et de l'enseignement - notamment au centre de création pédagogique Turbine à Montréal.

Ensemble, nous avons interrogé les passerelles et les clivages entre démarches scientifiques, pédagogiques et esthétiques. Ce que montrent les photographies de Fabien Desage, c'est la possibilité de penser la relation entre la recherche en sociologie de l'urbanisme et la pratique photographique autrement que comme un rapport de subordination ou d'illustration, autrement aussi que comme un détour ou une ellipse: comme une forme de pensée expérimentale qui participe de la même matière-monde à laquelle elle s'intéresse et qui donc a le pouvoir (et le devoir) de contribuer à la transformer. Comme ces enfants en plein élan d'un saut (rubrique "Échappées belles »), la photographie partage une portion de la courbe qu'est la recherche. Dans les deux cas, il faut un arrachement au déjà connu, pensé et vu pour imaginer du nouveau. Photographiant, le chercheur a donc moins changé d'activité qu'intégré des pratiques pour faire une recherche qui suscite aussi un plaisir esthétique - et une impulsion transformatrice des espaces urbains normalisés, qui cherchent à policer les corps.

\section{Aperçu}

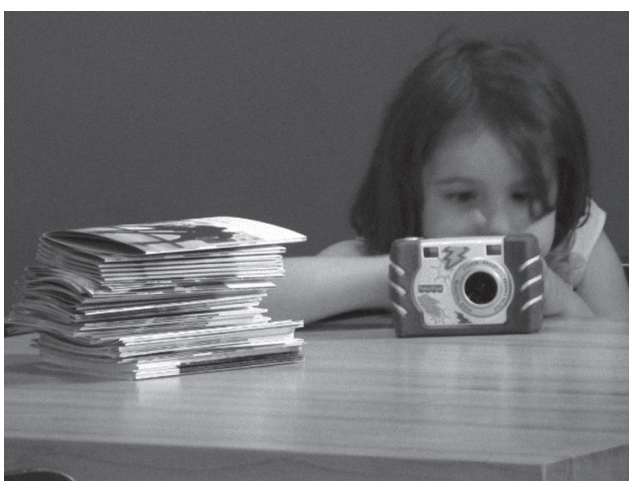

Fig. 7

Rencontre exposition avec Fabien Desage 


\section{Prolongation}

Les photographies ont été publiées avec un article cité ci-dessus dans le numéro Métropolitiques consacré aux enfants dans la ville, et qui continue à tisser le fil indisciplinaire: http://www.metropolitiques.eu/ Enfantillages-photographier-les.html

Les cinq rencontres indisciplinaires organisées à Montréal entre janvier et juin 2015 auront expérimenté une recherche que je dirais à présent " relationnelle ${ }^{24}$ " pour la différencier d'autres approches complémentaires (fondamentales, appliquées, spécialisées, ou érudites). En tant que titulaire de la Chaire d'études de la France contemporaine, j'y ai vu une manière de prendre appui sur la relation France-Québec pour ouvrir de nouveaux modes de rapport au lieu de rejouer les terrains de spécialisation et les rapports de force hérités. En amont des distinctions disciplinaires, des divisions entre types de connaissances et du clivage entre sciences dures ou molles, il s'agissait de connecter des formes de pensée et d'action en vertu de leur capacité de transformation du réel et des représentations. C'est une manière de pratiquer la recherche comme une force de reconfiguration et l'UniverCité comme un lieu de création permanente davantage qu'un espace d'archivage de savoirs morts - à la manière dont le DHC/ART transmue le white cube en kaléidoscope d'expériences vives.

Beaucoup reste à faire et à comprendre de cette expérience, à commencer par la forme à donner à une ressaisie critique. L'espace offert par ces «Carnets » aura été une magnifique opportunité I'aventure reste à poursuivre!

24 Au sens entendu par GLISSANT Édouard. Poétique de la relation. Paris: Gallimard, 1990. L'adjectif est employé en un sens différent dans le champ de l'art contemporain par BOURRIAUD Nicolas. Esthétique relationnelle. Paris: Les Presses du réel, 1998, p. 7. Pour une critique, voir BISHOP Claire. Artificial Hells. Participatory Arts and the Politics of Spectatorship. London and New York: Verso, 2012. 


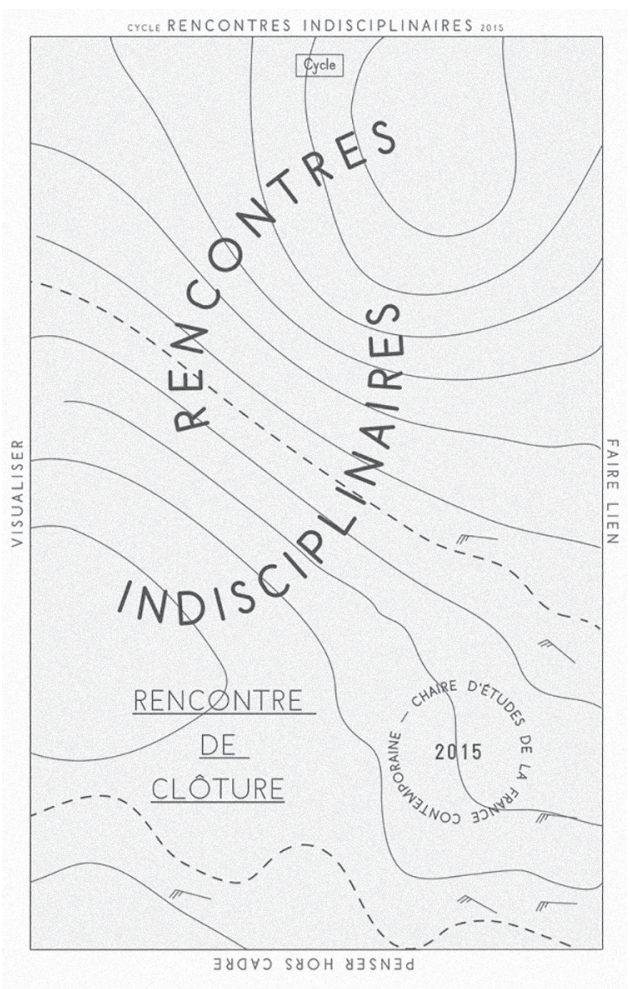

Nous espérons vous retrouver en nombre pour cette dernière rencontre du cycle indisciplinaire 2014-2015, qui se tiendra à la jonction des pratiques scientifiques et artistiques et dans le maillage d'un titulaire à une autre. Ce sera l'occasion de voir, d'entendre, d'échanger et de lever un verre - car la Chaire, décidément, n'est pas triste !

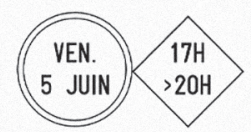

CAFE LE FIXE,

5985 rue Saint-Hubert,

Montréal (métro Rosemont)

\section{La question urbaine revisitée}

Enfantillages : enfants (et parents) dans l'espace public

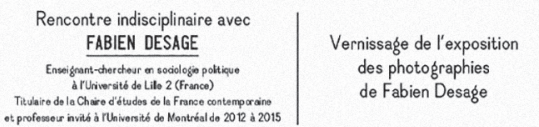

La recherche en sociologie ou en urbanisme peut-elle entrer La recherche en sociologie ou en urbenisme peut-elle entrer
en dialogue avec une pratique photographique? Et dans quelles lansues se oarient-elles alors?
La recriercile en socioluge ou eri urvernsmite peut-ente entrer entique photographique? Et dans quelles langues se parient-elles alors?

La présence dans l'espace public prescrit et proscrit un ensemble de comportements, attitudes, postures corporelles, manières de se mouvoir, d'interagir ou - le plus souvent - de s'éviter. Si l'espace public est donc avant tout facteur d'ordre, de pratiques réślées et prévisibles (de disciplines), le propos de cette exposition est notamment d'interroger la place singulière qu'y tiennent de ce point de vue les enfants. Plus souvent que les adultes, ces derniers semblent en effet susceptibles de "sortir des cadres" : par le jeu, l'indiscipline... mais aussi l'ennui, susceptibles de " sortir des cadres " : par le jeu, indiscipline... mais aussi lennui, doute au « photogénisme » prèté aux enfants, que cette exposition questionne et contextualise. (F.D.)

Fabien Desaģe pratique la photographie de rue depuis plusieurs années et s'intéresse notamment à la manière dont elle peut être mobilisée dans le cadre des sciences sociales et des pratiques militantes.

Participant-e-s: MARIE-PIERRE LABRIE Educatrice artistique et artiste, membre du Centre de création pédaǵoǵqque Turbine

Merci de confirmer votre présence à fabien.desaga@@gmail.com ou myriam.suchat@univ-paris3.fr

\section{VENT DOMINANT}

Un vent $d$ 'indiscipline souffle sur la Chaire d'études de la France contemporaine! De courants chauds en bourrasques, virevoltes et tourbillons, nous traverserons des contrées différentes - et pourtant moins éloiǵnées qu'il n'y paraît à la recherche d'espaces non encore cartoǵraphiés ou pas encore advenus. Chaque rencontre, imaginée en complicité avec les deux bords de l'Atlantique, mettra en jeu un format différent (atelier, pensée en marche, performance...) pour établir des relations et des circulations inédites. Donner des coups de sonde dans l'inconnu, faire lien et penser hors cadre : tel est le mandat d'une démarche indisciplinaire qui conçoit la recherche comme une forme de création.

Aucune connaissance disciplinaire ni académique n'est requise pour suivre ce cycle, qui invite à se réunir et à réunir en nous ce que nous avons de disponibilité à la pensée critique, à l'art, à l'écoute et à l'audace.

\section{Rencontres passées}

13 MARS 2015 - Atelier cartographique au Studio XX INTERVALLES $\cap$ INTERSTICES $\cap$ BATTEMENTS, RENDRE VISIBLES DES ESPACES-TEMPS A PARTAGER avec Pascal Nicolas-Lo Strat et Emmanuelle Jacques

24 AVRIL 2015 - Atelier de visualisation ǵraphique DONNER A VOIR UNE PENSEE QUI (SE) CHERCHE avec Samuel Tronçon (Ágence Résurgénce, France)

25 AVRIL 2015 - Atelier de traduction en languess signées (sur invitation) MONTRE-MOI TA LANGUE! avec Julie Châteauvert et Daz Sanders

25 MAl 2015 - Pensée en marche, visite déguidée et atelier de recherche-action-création AVENTURES DE LIGNES avec Dominic Hardy

Fig. 8 